\title{
TRANSFORMABLE STRUCTURES: MATERIALISING DESIGN FOR CHANGE
}

\author{
STIJN BRANCART, ANNE PADUART, ALINE VERGAUWEN, CAMILLE VANDERVAEREN, \\ LARS DE LAET \& NIELS DE TEMMERMAN \\ Department of Architectural Engineering, Vrije Universiteit Brussel (VUB), Belgium.
}

\begin{abstract}
Living in an age of rapid changes, designers are challenged to create solutions that remain sustainable in a continuously evolving environment. Since most of our earth's resources are finite, these solutions should incorporate efficient material use and reuse. Buildings and structures are always in transition. Facilitating these transformations is vital to the sustainable development of our built environment. With our group we study, develop and assess transformable structures on different scales, in different contexts and for various time-spans and purposes. This paper presents our work on transformable structures, based on four case studies: a kinetic curved-line folding component, a temporary and rapidly assembled structure, a dynamic wall assembly and a BIM tool for material flow assessment of adaptable buildings. Although varying in scale or purpose, these cases demonstrate the same key principles of transformability. Reducing the complexity of the connections and structural system facilitates an easy and rapid assembly, but also allows users and locals to participate in the assembly, maintenance, reconfiguration and deconstruction of the structure. Apart from benefits during the assembly and adaptability, it is important to assess transformable structures and building solutions on their material and cost effectiveness. With BIM tools it is possible to incorporate this assessment already in the conceptual design phases of a project, as illustrated in the fourth case.

Keywords: BIM, deployable structures, design for change, kit-of-parts structures, material flows, prototyping, transformable structures.
\end{abstract}

\section{INTRODUCTION}

The complex nature of our built environment subjects it to continuous evolutionary processes. The subsequent changes in cultural trends, global markets and technological innovation increasingly lead to resource depletion and waste production, and thus endanger the self-sustaining nature of our planet. Because most of the earth's mineral and fossil resources are finite, they should be used and reused wisely. Designers are challenged to create solutions that remain sustainable in a continuously changing context. The structures of the built environment in which we operate are never end states, but phases of a process. Facilitating transformations is vital to sustainable development. This requires holistic approaches that take change into account and help alleviate future problems.

By introducing transformational capacity at different design levels, we want to maximise the sustainability of settlements, structures and components through time while minimising the waste of resources. We believe that transformability can act as an important catalyst for sustainable development because of the social, economic and ecological qualities it generates over time and the life-cycle resource management it incorporates. With our research group, we study, analyse, design and assess transformable structures varying in scale, context, timespan and purpose.

This paper discusses the main principles of the development of transformable structures, illustrated by four case studies. These cases show the different methodologies and scale levels at which we can introduce transformational capacity to our built environment: a kinetic curved-line folding component, a rapid assembly kit for a temporary structure, a dynamic 
wall assembly and a Building Information Modelling (BIM) tool to assess the material flows in adaptable buildings.

Through these cases, the potential of transformable solutions becomes apparent for different scale levels and applications. Looking at structures over their complete lifecycle and designing them as part of a continuous process of transition, incorporates the functional and maintenance phases as well as the assembly, disassembly and post-processing in the design and increases their efficiency. Decreasing the technical complexity of the system, developing a manual assembly process, and incorporating local materials and expertise facilitate a participatory process and lead to social ownership and eventually to an increased longevity of the structure, which is better adapted throughout its functional lifetime.

\section{TRANSFORMABLE STRUCTURES FOR SUSTAINABLE DEVELOPMENT}

Thanks to its evolutionary character, nature has the intrinsic capacity to adapt to changing environmental conditions, leading not only to evolution over long periods of time but also to seasonal changes and resilience to ecological shocks. Contrary to natural elements, the manmade elements of our built environment often do not possess this adaptive capacity. Changes in the environment ask for large interventions with consequent waste production, material consumption, and economical and ecological costs. Transformable structures are designed to anticipate future changes and allow adaptation in a much more efficient way. This is certainly not a new concept. Nomadic tribes exploited the elasticity of local materials to create vernacular structures that could easily be disassembled, transported and reused, like the Mongolian yurt and the Madan mudhif [1]. All around us, common objects such as umbrellas or camping tents use principles of transformation to provide adaptability to changing needs and environments. With our research group we aim to develop these transformable systems for contemporary building solutions to increase the reuse potential of our building materials and decrease the production of waste.

\subsection{Two principles: deployability and design for disassembly}

Generally, we distinguish two principles for the design of transformable structures: deployability and design for disassembly.

Deployability allows the instantaneous opening or closing of a structure to transform it from a compact configuration to an expanded, functional space enclosure [2]. As with plants or even animals in nature, this volume or surface expansion allows capturing or protecting from the sun, the rain and other environmental factors. A well-known example is the deployable scissor structure, which relies on the rotation of simple, intermediately connected bars (see Fig. 1). Although the design and fabrication of a deployable structure can often become complex, due to the complexity of the nodes, the assembly process is relatively simple and can be performed by laymen. Deployable systems are very well suited for kinetic applications, such as façade shading devices and convertible roof structures.

The complexity of natural systems arises from interactions between a set of simple components. Likewise, kit-of-parts structures consist of a set of (often standardised) parts that can be assembled through basic rules or assembly modes. Design for disassembly entails using reversible connections to allow disassembly of the structure and reuse of the components [3]. The principle is applicable for small-scale temporary structures, but can also be applied (often to a lesser extent) to full-scale buildings and building elements. Thinking about possible reconfigurations can increase the reuse potential of the components in kit-ofparts structures. Avoiding complicated connections decreases the assembly time and allows 

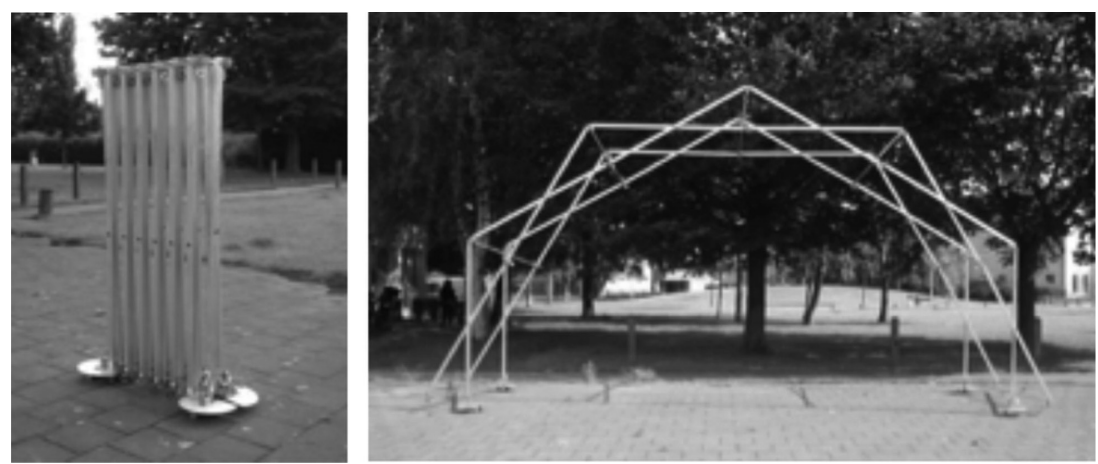

Figure 1: A deployable structure can instantaneously transform from a compact bundle of elements to a full-scale space enclosure. (L. Alegria Mira, A. P. Thrall and N. De Temmerman).

non-experts to assemble the structures manually, thus increasing the potential for user participation.

\subsection{Design for simplicity}

The basic premise of design for change is a systemic design approach aimed at developing structural and building systems that can be adapted with a minimum of expert intervention, giving the user the control to perform changes according to their needs. This calls for simple systems that can be manually erected, demounted and reconfigured. The aim is a participatory process in which the user is enabled to shape the built environment. As such, spontaneous and instantaneous changes can occur when needed. Moreover, transformable structures are well suited for temporary structures, supporting temporary activities and allowing citizens to reclaim abandoned or vacant sites for 'pop up' activities such as neighbourhood gardens or playgrounds [4]. This level of participation can only be achieved when the simplicity of the system allows laymen to operate it. In kit-of-parts structures this entails a limited set of simple components and a straightforward set of manual handlings (a user guide if you will). Deployable structures mainly require a simple actuation system, as expert labourers can still perform the pre-assembly. Moreover, striving for a uniformity of complexity throughout the structure ensures a well-balanced fabrication process.

\subsection{Assessment of transformable structures}

One of the main challenges for the implementation of design for change and transformable building solutions in the design and construction of our building stock is the high uncertainty regarding the actual environmental and financial costs and savings. In their doctoral dissertations, Mieke Vandenbroucke [5] and Galle [6] studied and developed tools for the life cycle assessment $\left(\mathrm{CO}_{2}\right.$ emission during the construction, functional lifetime and end-of-life) and life cycle costing (financial cost over the entire lifetime) of transformable building elements. Their analyses show that a higher initial cost can be overcome by decreased maintenance and refurbishment costs for transformable elements that are well placed and likely to be altered or removed during the building's lifetime. As such the studies validate the layering principle (see Fig. 2), which physically separates layers with different functional lifetimes. 


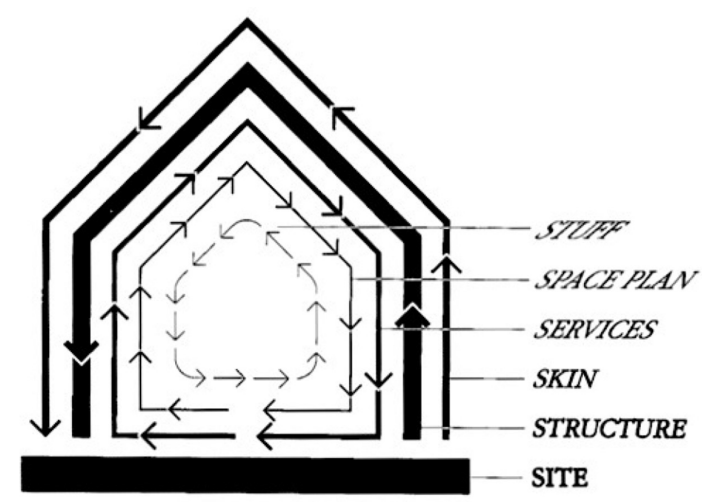

Figure 2: Physically separating building layers based on their functional lifetime, facilitates maintenance and replacement of the 'faster' ones [7].

\section{MATERIALISING DESIGN FOR CHANGE: CASES}

The next part discusses four case studies of transformable building solutions: a kinetic curved-line folding system, a temporary kit-of-parts structure, a dynamic wall assembly and a BIM tool for material flow assessment in adaptable buildings.

\subsection{Curved-line folding: design of a pliable component}

As mentioned before, a number of natural systems have developed modes of transformation to regulate and adapt to changing environmental conditions. Contrary to the complicated and mechanical way by which deployable structures are often developed, these natural systems are simple and elegant. Think of the curling of leaves or the opening of a flower. A number of these systems are what is called 'compliant' in mechanics. Howell [8] defines compliant mechanisms as 'something that is flexible to bend and in doing so accomplishes something useful'. It is the utilisation of the bending capacity of the materials and the deformation of the components that eliminates the need for complicated hinges and connections. As the act of folding a sheet of material along a curved line [9], curved-line folding translates well to compliant mechanisms. Contrary to its rigid counterpart origami, curved-line folding entails material deformation. Similar to the opening and closing of flowers, curved-line folding elements can act as one-degree-of-freedom mechanisms: deforming one piece results in the deformation of the entire structure [10], as shown in Fig. 3. This simplifies the actuation and makes these systems especially interesting for kinetic applications, such as the flectofin ${ }^{\circledR}$ shading device [11].

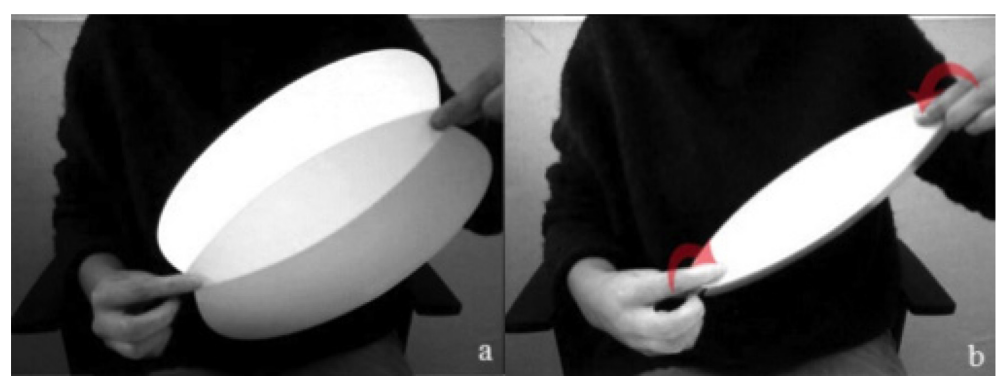

Figure 3: The deformation of the central spine controls the flapping of the whole [12]. 

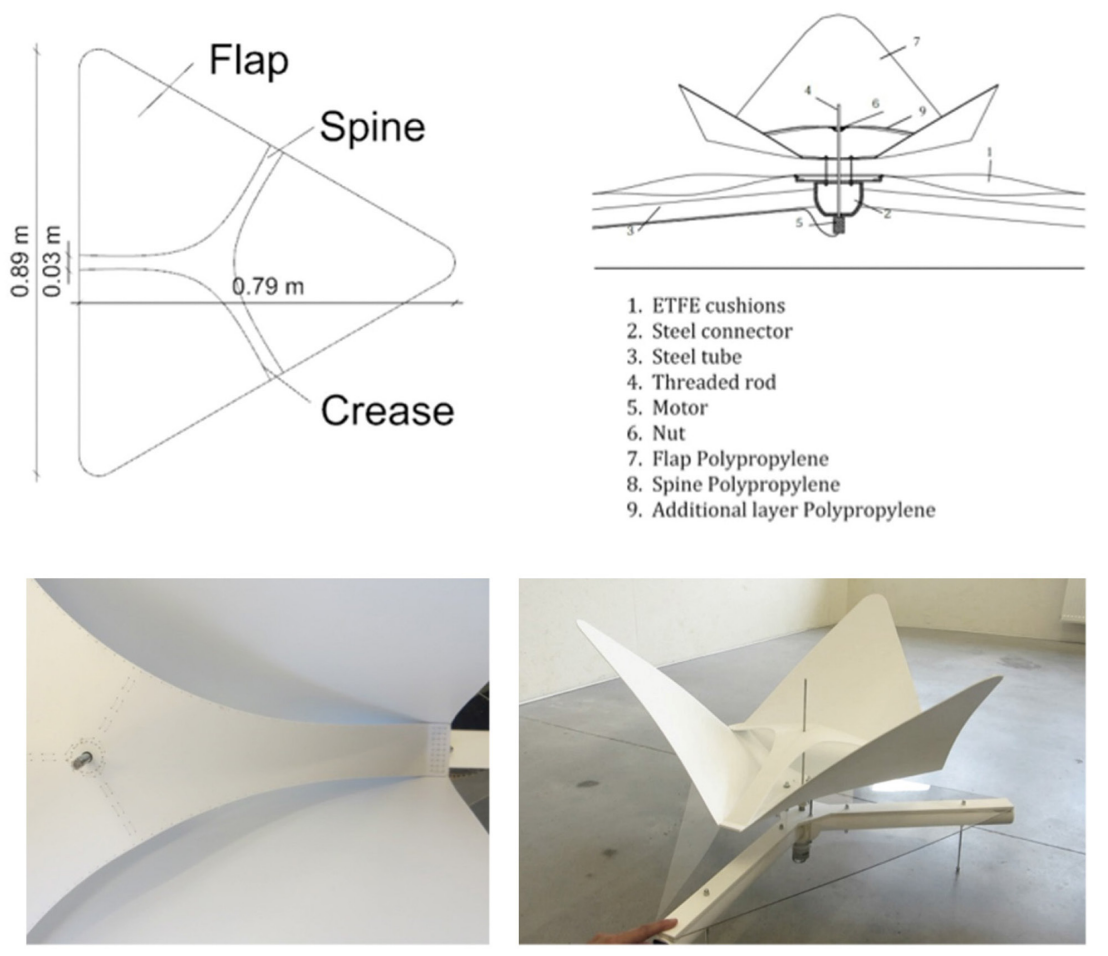

Figure 4: The double layering reduces the actuation of the curved-line folding component to moving the upper layer along a central threaded rod. (Adapted from [12, 13]).

When developing the principle of curved-line folding for kinetic applications, the aim should be to maintain this level of simplicity in the actuation of the system, avoiding sliders, robotic arms and other mechanical components. Therefore, we developed a system of double layering that transfers the deformation of the central spine (and consequently of the entire component) to a simple movement along a central axis. Figure 4 shows a prototype of such a system. By moving it along the threaded axis, the upper layer controls the incremental deformation of the entire system.

\subsection{UNDULATUS: a rapid assembly kit of self-interlocking, curved-line folding components}

For an exposition at the Muziekgebouw aan 't Ij in Amsterdam in 2015, we developed the Undulatus pavilion, a rapid assembly kit composed of curved-line folding components [14]. The main aims of its design were a rapid, manual assembly and disassembly, a compactly transportable stacked volume and the potential of extensive reuse. Using curved-line folding with flexible materials in a kinetic way allowed us to create 3D components from flat sheets in a fully reversible assembly. Contrary to the previous case study, the kinetic components are configured in a static structure.

The structure, composed of 99 polypropylene components, weighed less than 30 kilograms and could be transported in a very compact, flat-stacked package (see Fig. 5a). The use of integrated connections (Fig. 5b) facilitates an easy, step-by-step assembly process (Fig. 5c). The self-interlocking of the components (each component is locked by its connection to six 

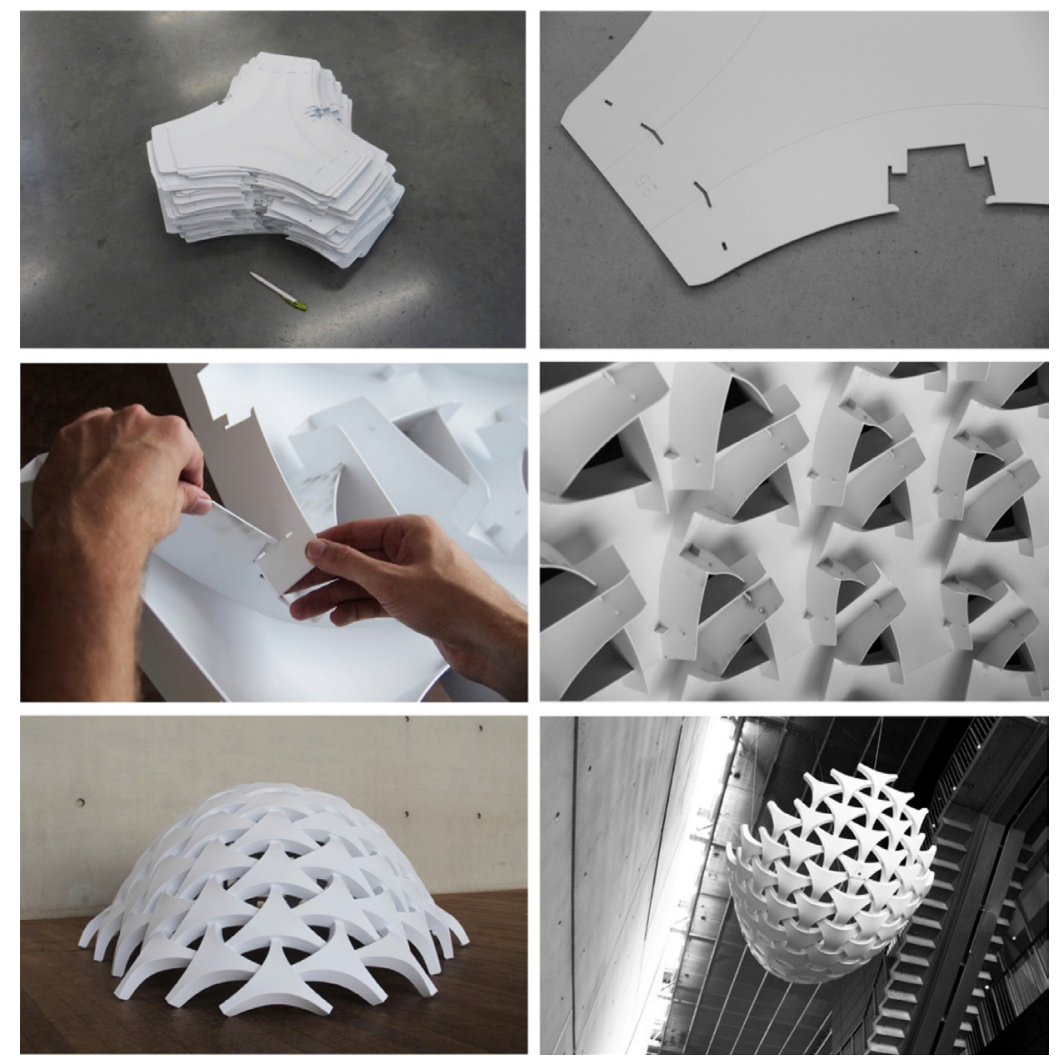

Figure 5: The Undulatus structure was developed as a compact kit of curved-line folding components (a). Thanks to simple, integrated connections (b) the structure could easily and rapidly be assembled (c) into a lightweight polypropylene structure without any additional components (d). The self-interlocking system results in a self-supporting shell structure (e) that was suspended for the exposition (f) [14].

surrounding elements) eliminates the need for additional components, contributing to the simplicity and lightweight nature of the structure (Fig. 5d). The structure shows a strong shell-like behaviour and is fully self-supporting (Fig. 5e), but was suspended for the exposition (Fig. 5f). Designed for rapid erection, this system allowed an assembly time of only 2 hours. The disassembly took only 15 minutes and the structure was reassembled after transportation to another location. Some plastic deformation occurred at the curved hinge line, but the components could still be packed in the original compact package.

A fully integrated parametric model contains the entire design process of the Undulatus structure. Starting from a target surface and a triangulated mesh (each point has a valency of six to allow the hexagonal compositions of the components) an interactive design workflow allows customising the structure and plotting all the components with integrated connections, ready for lasercutting (see Fig. 6). Markings on the components facilitate an organised assembly (which becomes like making a giant 3D puzzle). As such the complexity of the structure is concentrated in the design phase (development of the system and generation of the parametric model) permitting both experts and laymen to fabricate and assemble the structure. 


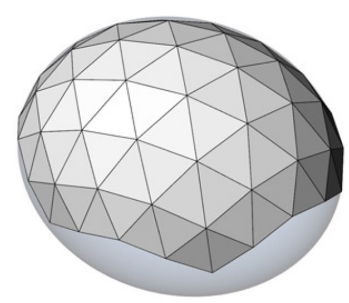

(a)

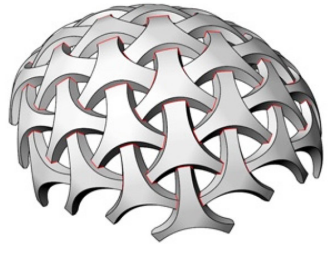

(b)

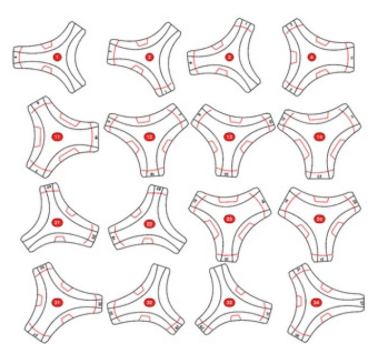

(c)

Figure 6: The complexity of the design of the Undulatus structure is contained in the parametric modelling, allowing a simple design workflow from triangulated target surface (a) to customisable 3D structure (b) and finally a plot of the components with integrated connections (c) [14].

\subsection{DynamicWall: an adaptable and reusable wall concept}

Since the construction sector is responsible for a large part of the material use and waste production, a high potential exists for the reuse and recycling of materials during building transformations and refurbishments. To allow this, we need to anticipate future alterations in the design and materialisation of our building stock [15]. The DynamicWall, conceptualised for the DynStra project within the framework of the 'Brussels Retrofit XL' platform (Innoviris), is a proof-of-concept of a transformable and multifunctional wall assembly. The wall system can include multiple functions (storage, technical appliances, etc.) and, through the use of reversible connections, facilitates component reuse and ease of assembly (see Fig. 7).

Since multiple prototypes were constructed and tested for their impact resistance and acoustic performance, the DynamicWall serves as a proof-of-concept not only for the transformational capacity of the system, but also for the performance and applicability of demountable building elements in the current building practice.

A demonstrator of the wall assembly was built in an architect's home in the Brussels-Capital Region (see Fig. 8). The system serves as a new partition wall that subdivides a large living room in a new bedroom and office space. Consisting of steel U-profiles, 3D connectors and suspended plywood panels, the wall provides separation as well as additional storage space. Its reversible design ensures that the wall can be adapted or removed (in reusable parts) leaving only small screw marks on the floor and ceiling.
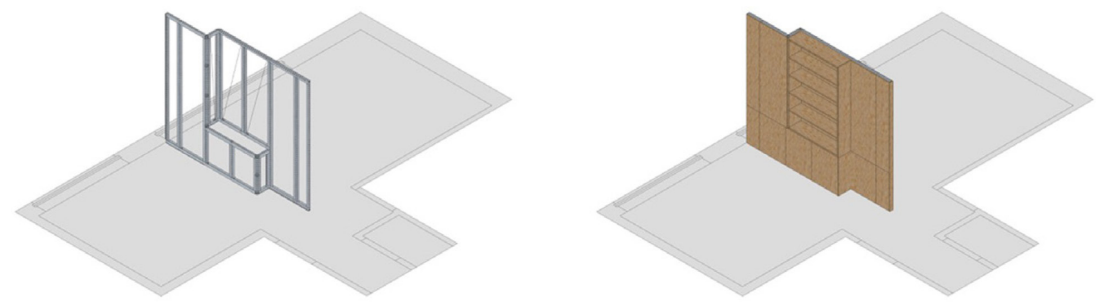

Figure 7: Thanks to reversible connections, the DynamicWall facilitates component reuse and ease of assembly. Acoustic and impact resistance tests show that the system is applicable in the current building practice. 

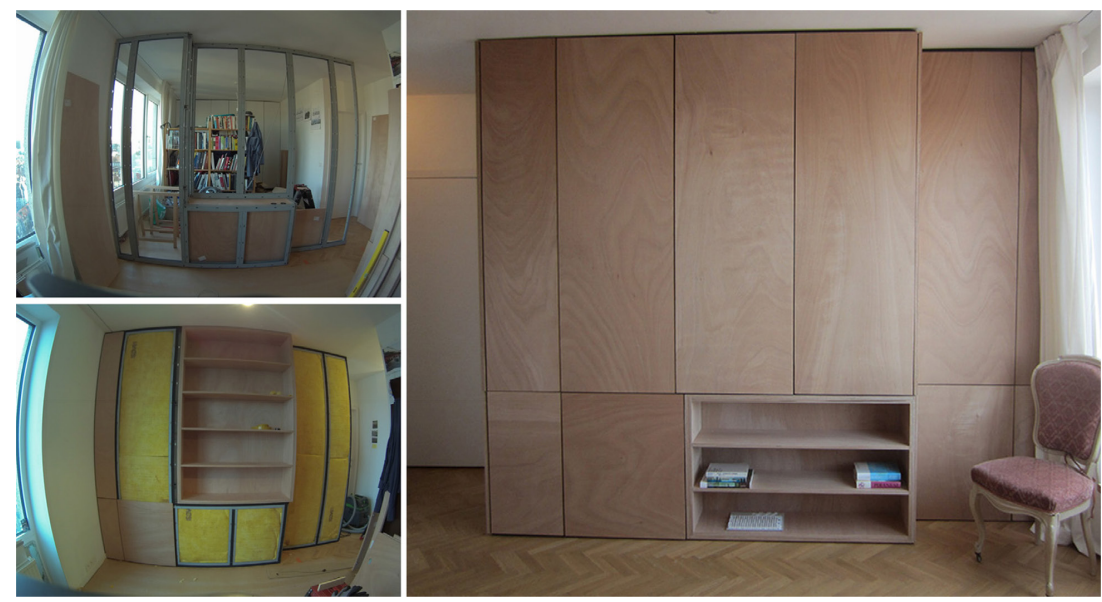

Figure 8: A demonstrator of the DynamicWall was constructed in an architect's home in Brussels. The wall subdivides a large living room in bedroom and office space, and is completely removable, consisting entirely of reusable parts. After deconstruction, only a few small marks will remain on the floor and ceiling.

\subsection{MATRx: a BIM tool for material flow assessment of adaptable buildings}

Although some successful implementations of Design for Disassembly exist, its application in the current building practice is often restrained by uncertainties regarding its global benefits and financial viability. Designers need accessible tools to quickly assess the impact of transformable building solutions on the material consumption, cost and transformational capacity of their project over its entire life cycle. MATRx is a tool that performs scenario-based assessments of a building's capacity to recover materials for reuse [16]. As such it allows designers to consciously reduce the overall material consumption of their buildings by making more informed choices. By integrating the reversibility of the connections and the durability of the materials in the conceptual design model, MATRx allows assessing the potential for reuse (see Fig. 9), thus reintegrating existing materials in the life-cycle of a

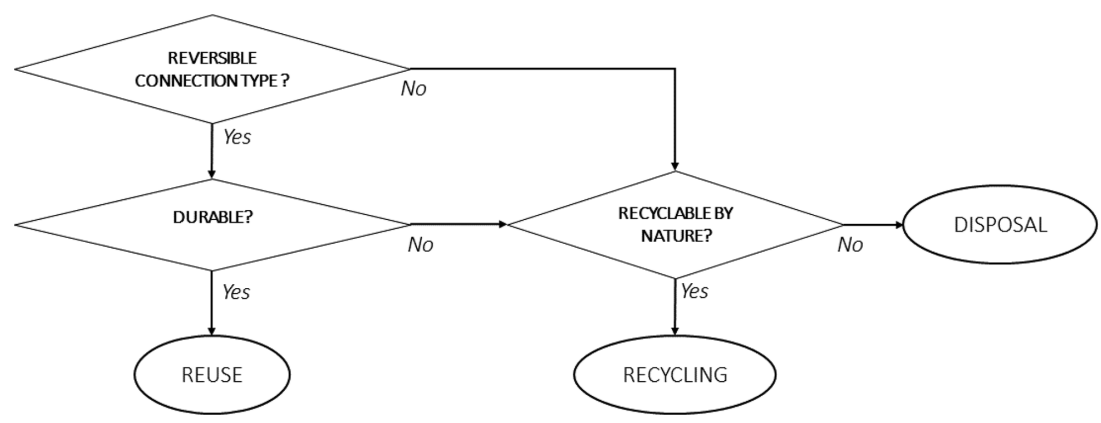

Figure 9: This simplified algorithm sorts the materials according to their reuse scenario. The tree main parameters used for assessing adaptability are: connection types, suitability for reuse (durability) and recycling [16]. 


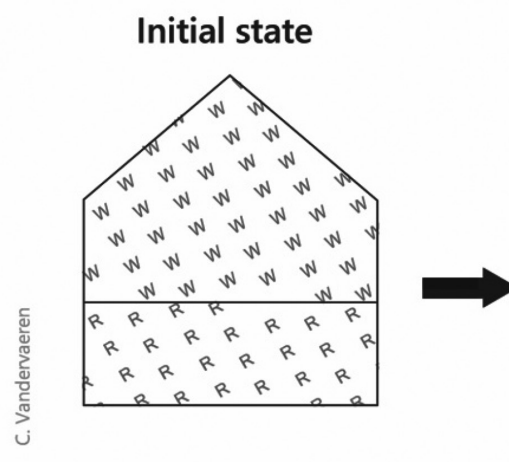

\section{During renovation}

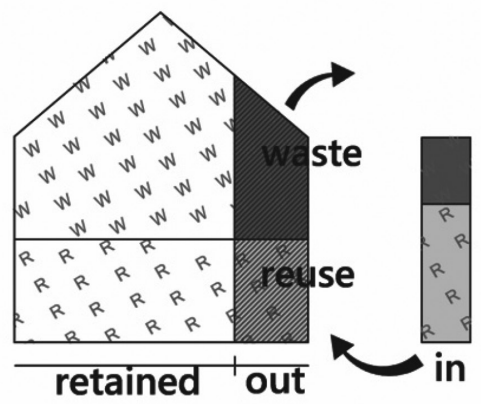

Figure 10: The MATRx assessment is based on a Material Flow Analysis. Among the materials evacuated through the renovation works, the ones that present a reuse potential (' $R$ ') can be relocated in the building instead of using new materials [16].

building and reducing the amount of recycled and disposed material. By playing on the durability of building materials, components can be reintegrated in the construction process, not only avoiding waste but also reducing the material consumption and consequent depletion of our earth's finite resources.

The MATRx tool is based on a Material Flow Analysis, integrating material flows in the design model of buildings (see Fig. 10). Implemented in the BIM software Revit ${ }^{\circledR}$ and the parametric modelling tool Dynamo, the tool has a low threshold for designers and engineers, allowing immediate integration in their day-to-day workflow. If supplemented by information on the life cycle cost and environmental impact of the material, tools like MATRx can provide architects and engineers with a global assessment of the impact of transformable solutions on their design projects already from the early design stages.

\section{CONCLUSIONS}

The three projects that were briefly described in this paper show the wide variety of methods and scale levels at which the principle of transformability can be implemented in our design practice and built environment. Thinking about the reuse of structures and components can in so many ways contribute to a decrease in waste production and facilitate adaptability and 'adaptedness' of a structure or building to its ever changing user needs. To ensure this user comfort and stimulate the user's participation designers need to be able to make better-informed choices during the design of their projects. Assessment tools based on Material Flow Analysis, Life Cycle Assessment and Life Cycle Costing can help them do this and can increase the involvement of non-experts in the development of transformable building solutions.

Ranging not only in scale, but also in the time over which they are expected to change, the cases in this paper show how the concept of transformability can be translated to specific design projects in varying contexts. Although the final solutions may vary, the aim for ease and speed of assembly, and simplicity and user involvement remain common denominators. 


\section{ACKNOWLEDGEMENTS}

This research was funded by the Flemish Agency for Innovation through Science and Technology (IWT), the Brussels Retrofit XL platform of Innoviris and the Vrije Universiteit Brussel (VUB).

\section{REFERENCES}

[1] Lienhard, J., Alpermann, H., Gengnagel, C. \& Knippers, J., Active bending, a review on structures where bending is used as a self-formation process. International Journal of Space Structures, 28(3), pp. 187-196, 2013.

[2] Hanaor, A. \& Levy, R., Evaluations of deployable structures for space enclosures. International Journal of Space Structures, 16(4), pp. 211-229, 2001. https://doi.org/10.1260/026635101760832172

[3] Debacker, W., Henrotay, C., Paduart, A., Elsen, S., De Wilde, P. \& Hendrickx, H., Fourdimensional design: from strategies to cases - generation of fractal grammar for reusing building elements. International Journal of Ecodynamics, 2(4), pp. 258-277, 2008. https://doi.org/10.2495/ECO-V2-N4-258-277

[4] Bishop, P. \& Williams, L., The Temporary City. Routledge: Abingdon, 2012.

[5] Vandenbroucke, M., Design, dimensioning and evaluation of demountable building elements. Doctoral thesis, Vrije Universiteit Brussel, Brussels, 2016.

[6] Galle, W., Scenario based life cycle costing, an enhanced method for evaluating the financial feasibility of transformable building. Doctoral thesis, Vrije Universiteit Brussel, Brussels, 2016.

[7] Brand, S., How Buildings Learn, What Happens After They're Built, Quebecor Printing, Tennessee, 1995.

[8] Howell, L.L., Compliant Mechanisms, John Wiley \& Sons: New York, 2001.

[9] Demaine, E.D., Demaine, M.L., Koschitz, D. \& Tachi, T., Curved crease folding a review on art, design and mathematics. Proceedings of the IABSE-IASS Symposium: Taller, Longer, Lighter, London, UK, 2011.

[10] Vergauwen, A., Curved-line folding, exploring, understanding and designing pliable structures for kinetic architecture. Doctoral thesis, Vrije Universiteit Brussel, Brussels, 2016.

[11] Lienhard, J., Schleicher, S., Poppinga, S., Masselter, T., Milwich, M., Speck, T. \& Knippers, J., Flectofin: a hingeless flapping mechanism inspired by nature. Bioinspiration \& Biomimetics, 6(4), 2011. https://doi.org/10.1088/1748-3182/6/4/045001

[12] Vergauwen, A., De Laet, L. \& De Temmerman, N., Computational modelling methods for pliable structures based on curved-line folding. Computer-Aided Design, 83, pp. 51-63, 2017. https://doi.org/10.1016/j.cad.2016.10.002

[13] Roger, A., Bending-activated transformable façade and roof elements. Master thesis, Vrije Universiteit Brussel, Brussels, 2014.

[14] Brancart, S., Vergauwen, A., Roovers, K., Van Den Bremt, D., De Laet, L. \& De Temmerman, N., UNDULATUS: design and fabrication of a self-interlocking modular shell structure based on curved-line folding. Future visions, Proc. intern. symp., Amsterdam, 2015.

[15] Paduart, A., Re-design for change: A 4 dimensional renovation approach towards a dynamic and sustainable building stock. Doctoral thesis, Vrije Universiteit Brussel, Brussels, 2012.

[16] Vandervaeren, C., Development of a BIM tool for material flow assessment of adaptable buildings. Master thesis, Vrije Univeristeit Brussel, Brussels, 2016. 